Cerebrovasc Dis 2006;22:284-285

DOI: 10.1159/000094331

\section{Abducens Nucleus Syndrome due to Pontine Haemorrhage}

\section{F. Lhermitte, M. Pagès}

Department of Neurology, Centre Gui de Chauliac, Montpellier, France

\section{Introduction}

Abnormalities of horizontal gaze are common in strokes involving the caudal part of the pons and generally associated with motor, sensory, cerebellar and cranial nerve dysfunction. Isolated abducens nerve palsy is rare $[1,2]$ and due to a small lesion which affects the radicular fascicles of the 6th cranial nerve and spares the abducens nucleus [3]. Conversely, abducens nucleus lesions produce a conjugate paralysis [4] but not an isolated lateral rectus muscle palsy.

We report a case of unilateral conjugate gaze paralysis due to a small pontine haemorrhage involving the abducens nucleus which turned into a paralysis of the lateral rectus muscle.

\section{Case Report}

A 69-year-old patient, with a history of non-insulin-dependent diabetes mellitus, hypertension and hypercholesterolaemia, was admitted for headaches, vertigo and diplopia of acute onset which had been lasting for 3 days. A right peripheral facial paresis was noticed on the second day of the stroke. On admission, physical examination showed a complete paralysis of abduction of the right eye and adduction of the left eye resulting in a paralysis of conjugate gaze towards the right side. Both saccades and pursuit movements were involved. There was no strabismus and both eyes were in the midline at rest. Vertical eye movements (saccades and pursuit) and convergence were normal. There was neither pupillary abnormality nor cranial nerve deficit. A rightward gait deviation was observed. CT scan and MRI revealed a small haemorrhage measuring $6 \mathrm{~mm}$, located in the right paramedian pontine tegmentum (fig. 1 and 2).

In 9 days, ophthalmological symptoms improved so that the patient only presented with a paralysis of abduction of the right eye. Left eye movements were clinically normal. Gait disturbances recovered more slowly. Three months later, the patient still had a paralysis of the right lateral muscle, without any other symptom. MRI performed 7 months later showed a small and old haemorrhagic lesion in the right pontine tegmentum.

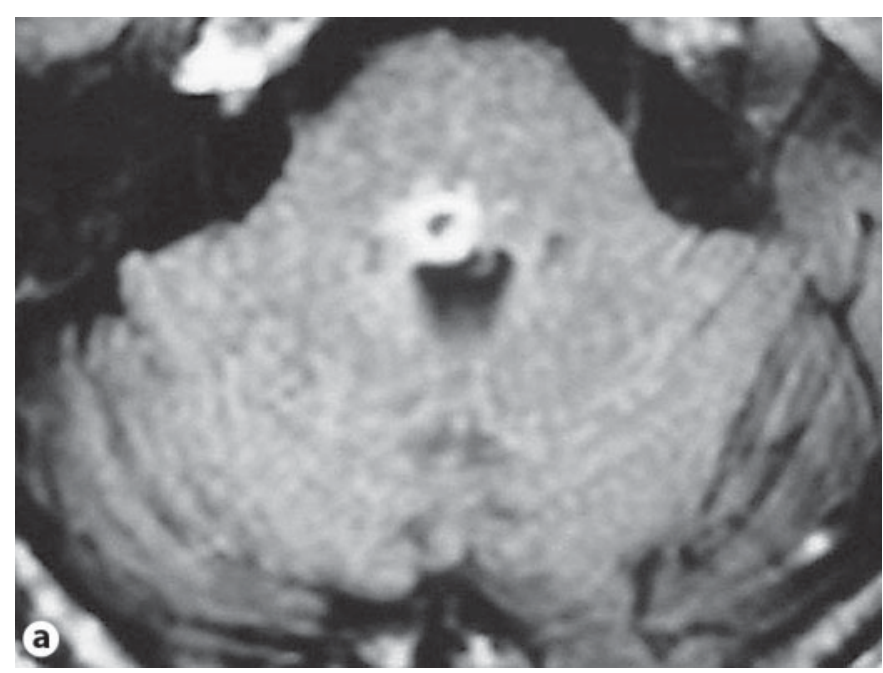

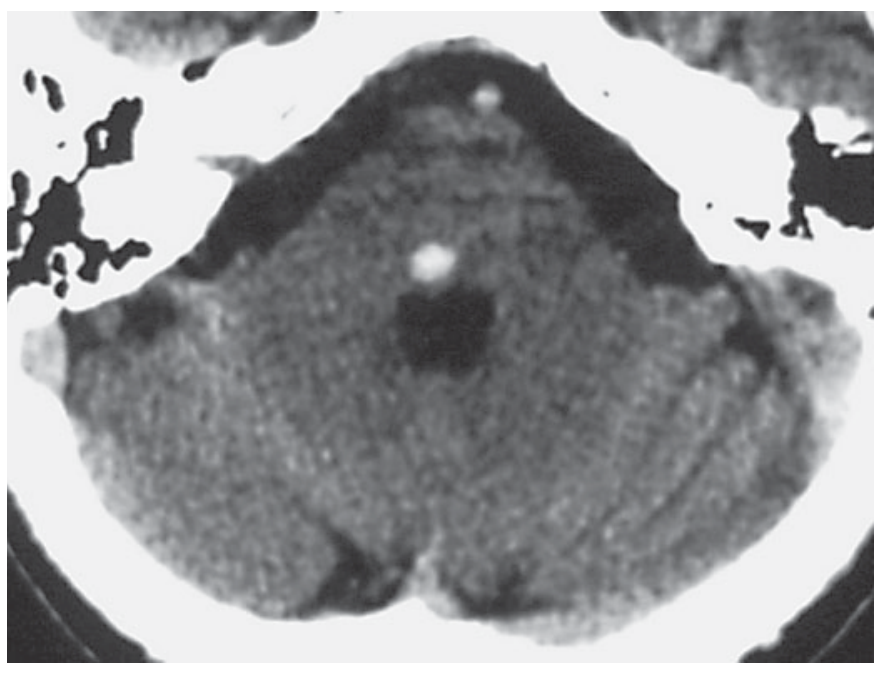

Fig. 1. CT scan. Spontaneous hyperdensity located in the right pontine tegmentum.

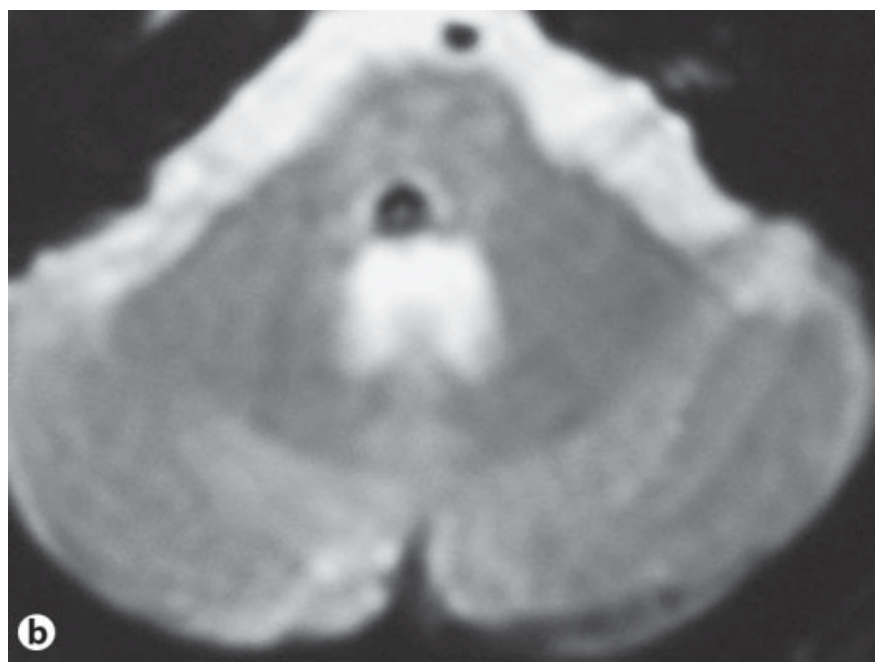

Fig. 2. MRI, FLAIR sequence (a) and $T_{2}{ }^{*}$-weighted sequence (b). Slight compression of the wall of the 4 th ventricle by the haematoma. 


\section{Discussion}

Our patient presented with a small spontaneous haematoma of the right pontine tegmentum which was revealed by vestibular syndrome, ophthalmoplegia and transient right facial palsy. A conjugate gaze paralysis toward the right side was observed during the first days and quickly turned into a paralysis of the lateral rectus muscle. The course of the disease and the neuroradiological findings suggest an involvement of the right abducens nucleus.

The abducens nucleus is the final common pathway for horizontal conjugate eye movements $[4,5]$. It contains 2 populations of neurons: motor neurons, which innervate the ipsilateral rectus muscle, and interneurons, which are connected with the contralateral nucleus of the medial rectus muscle after crossing the midline, via the medial longitudinal fasciculus. Lesions of the 6th nucleus produce paralysis of abduction of the ipsilateral eye and of adduction of the contralateral eye, that is an ipsilateral gaze palsy [6]. Isolated abducens nucleus syndrome is exceedingly rare, because the lesion must be strictly limited to this small area without extending to the surrounding structures. Due to the looping of the 7 th cranial nerve fascicle around the abducens nucleus, a peripheral facial palsy may be associated with the conjugate gaze palsy [4]. A few cases have been reported, including infarctions, histiocytosis $\mathrm{X}$, tumours, multiple sclerosis and Wernicke-Korsakoff syndrome $[4,6-8]$. To our knowledge, there is no case due to brain haemorrhage.

Involvement of the caudal paramedian pontine reticular formation, which is the premotor structure for horizontal saccades, may also produce an ipsilateral gaze palsy, but pursuit and vestibuloocular reflexes are preserved and ipsilateral saccades, including those in the contralateral hemifield, are lacking [5]. In our case, the only clinical finding which supports the hypothesis of abducens nucleus involvement is the normal pursuit movements, but they are always difficult to interpret. Oculographic studies were not performed and we do not know whether the velocity of the saccades from the left position to the midline was normal or not; thus, from a clinical point of view, the location of the lesion on the oculomotor pathway - abducens nucleus, paramedian pontine reticular formation or both of them - is uncertain. However, MRI clearly shows that the haematoma involved the area of the abducens nucleus, with a slight compression of the wall of the 4 th ventricle. The paramedian pontine reticular formation, which is in close contact with the nucleus, is located a little more anteriorly and superiorly so that it seems to be spared by the haematoma. Finally, an isolated lesion of the paramedian pontine reticular formation may be ruled out by MRI findings, and an isolated involvement of the abducens nucleus seems more likely than a lesion of both structures.

In our patient, an isolated palsy of the lateral rectus muscle followed the initial conjugate palsy. A faster improvement of the limitation of adduction compared with that of abduction has already been observed [7]. It has been suggested that the motor neurons in the abducens nucleus could be more sensitive to injury than the interneurons [3], but this hypothesis seems unlikely in case of haematoma. It may be supposed that the haemorrhage mainly involved the fascicular origin of the 6th cranial nerve in the abducens nucleus and that nuclear damage was due to a compression effect, in the same way that the initial vestibular nucleus and 7 th cranial nerve were involved.

\section{References}

1 Donaldson D, Rosenberg NL: Infarction of abducens nerve fascicle as cause of isolated sixth nerve palsy related to hypertension. Neurology 1988;38:1654.

2 Fukutake T, Hirayama K: Isolated abducens nerve palsy from pontine infarction in a diabetic patient. Neurology 1992;42:226.

3 Bronstein AM, Morris J, Du Boulay G, Gresty MA, Rudge P: Abnormalities of horizontal gaze: clinical, oculographic and magnetic resonance imaging findings. I. Abducens palsy. J Neurol Neurosurg Psychiatry 1990; 53:194-199.

4 Müri RM, Chermann JF, Cohen L, Rivaud S, Pierrot-Deseilligny C: Ocular motor consequences of damage to the abducens nucleus area in humans. J Neuroophthalmol 1996;16:191-195.

5 Pierrot-Deseilligny C: Circuits oculomoteurs centraux. Rev Neurol 1985; 141:349-370.

6 Pierrot-Deseilligny C, Goasguen J: Isolated abducens nucleus damage due to histiocytosis X. Brain 1984;107:1019-1032.

7 Saitou N, Takahashi J, Kashima Y, Ishikawa H: Horizontal gaze palsy due to lesion of the abducens nucleus detected with magnetic resonance imaging. Nippon Ganka Gakkai Sasshi 1995;99:624-627.

8 Hamasaki S, Motomura M, Koga H, Nakamura T, Yoshimura T: Persistent lateral gaze palsy and abducens nerve palsy due to pontine infarction. Rinsho Shinkeigaku 1998;38:344-346.

Prof. Michel Pagès Service de Neurologie, Centre Gui de Chauliac 80 Avenue Fliche, FR-34295 Montpellier Cedex 5 (France)

Tel. +3346733 73 63, Fax +33467337980

E-Mailm-pages@chu-montpellier.fr 